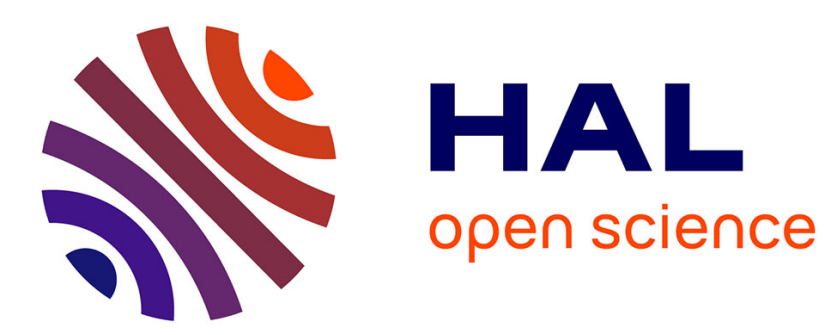

\title{
Habiter à Dûwîqa au CaireDedans et dehors d'une société de proximité
}

Nicolas Puig

\section{To cite this version:}

Nicolas Puig. Habiter à Dûwîqa au CaireDedans et dehors d'une société de proximité. Autrepart Revue de sciences sociales au Sud, 2003, 25, pp.137-152. halshs-00003943

\section{HAL Id: halshs-00003943 \\ https://shs.hal.science/halshs-00003943}

Submitted on 11 Jul 2005

HAL is a multi-disciplinary open access archive for the deposit and dissemination of scientific research documents, whether they are published or not. The documents may come from teaching and research institutions in France or abroad, or from public or private research centers.
L'archive ouverte pluridisciplinaire HAL, est destinée au dépôt et à la diffusion de documents scientifiques de niveau recherche, publiés ou non, émanant des établissements d'enseignement et de recherche français ou étrangers, des laboratoires publics ou privés. 


\title{
TITRE COURANT : \\ PAIR : Nicolas Puig \\ IMPAIR : Habiter à Dûwîqa au Caire
}

\section{Habiter à Dûwîqa au Caire, les paradoxes d'une zone d'habitat temporaire}

\author{
Nicolas Puig * \\ * Chargé de recherches, IRD (UR013), Cedej. \\ Autrepart (25), 2003 :
}

Habiter à Dûwîqa, c'est forcément venir d'ailleurs, pourrait-on écrire paraphrasant une formule de Jacques Berque ${ }^{1}$ appliquée au refus de l'autochtonie dont font montre certaines populations du Maghreb. La zone, appartenant au district de Minshât Nasîr, est située dans la proche périphérie est du Caire et s'urbanise à partir de la fin des années soixante-dix. Elle est constituée, en partie, de petits appartements de deux pièces appelés «refuges temporaires» selon la terminologie officielle et alloués par les services du gouvernorat du Caire à des familles en situation précaire en provenance majoritairement des quartiers de la vieille ville (Le Caire fatimide fondé au $\mathrm{X}^{\mathrm{e}}$ siècle). Elle comprend pour le reste des habitations construites sans autorisation administrative préalable. Le quartier est géographiquement proche des centres historiques cairotes, la vieille ville et la ville européenne érigée au XIX ${ }^{\mathrm{e}}$ siècle par le Khédive Ismaïl. Mais, au regard de la représentation dominante de la légitimité citadine, il s'agit d'un autre monde ${ }^{2}$. Vu des quartiers centraux, Dûwîqa ne semble pas vraiment appartenir à la ville telle qu'elle est vue des quartiers centraux.

Toutefois, malgré ces handicaps et en dépit du caractère temporaire de la résidence l'ensemble des occupants devrait être relogé dans un habitat de meilleure qualité -, une vie collective s'est développée, rappelant celle des quartiers historiques. Nous verrons qu'elle se caractérise par une activité sociale ouverte et soumise au contrôle intégrateur du voisinage.

En parallèle, les déplacements dans la ville se révèlent importants. Ces pratiques de mobilité quotidienne constituent, dans le cas de ménages paupérisés, une ressource de leur pratique de la ville. Elles contribuent également à intensifier les expériences urbaines en multipliant les parcours et en développant ainsi, chez ces citadins stigmatisés, les «compétences de rassemblement» que les habitants du centre-ville et des quartiers chics pensent seuls détenir ${ }^{3}:$ « Le phénomène urbain comme mode de vie, c'est précisément cet accès problématique aux modes de vie et aux langages de l'autre. Et c'est, par là même, le développement, pour ceux qui ont à vivre en mitoyens tout en

\footnotetext{
1 Jacques Berque avait eu cette formule à propos de l'Atlas marocain où « être de quelque part, c'est venir d'ailleurs ».

La représentation de base qui distingue quartier chic et quartier populaire au Caire s'est enrichie depuis les années soixante-dix d'un terme supplémentaire: informel ou non réglementaire ('ashwâ' $\hat{)}$ ). Ces « fonds de la ville » correspondent aux nouveaux quartiers péricentraux et périphériques tel Minshât Nasîr [Labib, Battain, 1991].

3 La synthèse réalisée sur ce sujet par Patrick Haenni [2001: 30-32] remet en cause la thèse diffuse en Égypte « d'une extranéité radicale des "cultures de pauvreté" vis-à-vis des structures de l'État et de la ville comme mode de vie ».
} 
négociant le sens commun de l'intervalle qui les sépare, de compétences qui ne sont plus simplement des compétences d'appartenances (des compétences de membres) mais des compétences de rassemblement » [Joseph, 1997 : 136].

C'est ainsi, par le double prisme de son intégration dans un ensemble communautaire et de ses mobilités quotidiennes en direction des autres quartiers de la ville que je souhaite rendre compte de deux facettes de l'expérience urbaine de la population de Dûwîqa, ce que l'on pourrait appeler les «dedans » et les "dehors » d'une société de proximité.

\section{Citadin local et citadin universel : approche et méthode}

Comment décrire les sociétés urbaines réputées pour leur tessiture communautaire ${ }^{4}$ en respectant deux points de vue : les « ancrages » territoriaux de leurs membres et les « passages » qu'ils effectuent en direction de différents points de la ville. La sociologie des espaces publics se concentre sur les situations au cours desquelles des citadins de diverses origines sont mis en coprésence et sur l'ordre qui naît de ces croisements fugaces. Les représentations du monde que les citadins en question tiennent de leurs appartenances et de leur inscription dans un milieu social ne sont pas retenues comme pertinentes par ce courant d'analyse. Ce dont il est rendu compte est plutôt la logique de la situation et la façon dont elle prescrit des comportements dans les relations sociales. "L'ethnographie des espaces publics met ainsi en scène une vision de la ville différente et complémentaire de la mosaïque de territoires élaborée à partir des monographies de quartiers » [Joseph, 1996: 110]. L'image emblématique de l'étranger y est à la fois "figure et analyseur de la mobilité urbaine», elle est le symbole de la condition citadine [Grafmeyer, Joseph, 1990 : 11]. Elle renvoie aux relations entre anonymes caractéristiques des expériences urbaines.

Inversement, une sociologie plus «localiste» s'intéresse aux communautés de quartier et tente de rendre compte des enracinements, voire des «traditions 5 » qui demeurent présentes au cœur même de la grande ville. Dans ces territoires familiers prennent place quotidiennement de solides sociabilités tandis que dans les « passages ", le fait de se rendre d'un lieu à un autre, se joue une part non négligeable de l'expérience urbaine. Dans le déplacement se forme une «culture de la diversité proche et de la distance radicale, qui fabrique de l'accessibilité et de la visibilité mutuelle sans échange » [Joseph, $1996: 115]$. Les mobilités quotidiennes, bien qu'étant contraintes dans une large mesure, constituent des moments de «déprise» des milieux de l'interconnaissance. Elles sont constitutives des expériences urbaines. De ce fait, la description de la réalité résidentielle et sociale du quartier doit s'accompagner d'une analyse des mobilités de ses habitants ${ }^{6}$.

\footnotetext{
4 S'agissant du Caire, dans un article qui demeure d'actualité, J.-Ch. Depaule [1990: 131] distingue plusieurs structurations de l'espace quotidien : d'une part, les «territoires d'une vie sociale ouverte que règle un contrôle collectif, intégrateur et exclusif », d'autre part, un mode dans lequel les habitants aspirent à vivre « portes fermés ».

$5 \quad$ Les analyses de Oscar Lewis sont emblématiques de ces approches [Hannerz, 1983 : 98 ].

6 Un troisième volet serait l'analyse des situations d'interactions dans les espaces publics et du rapport à la centralité des habitants des quartiers spécifiques. Je réserve cela à des développements ultérieurs, considérant que ce type d'analyse n'a pas sa place dans une publication traitant des dynamiques résidentielles.
} 
La méthode d'investigation tient compte de ce double objectif. Elle consiste d'une part en un programme d'observation in situ. Une partie des informations empiriques est issue d'une enquête ethnologique plus vaste conduite depuis trois années, qui porte sur l'organisation sociale de Darb al-Ahmar (dans Le Caire fatimide) et Dûwîqa et sur les cultures ${ }^{7}$ populaires dans la ville. Le recueil des données anthropologiques résulte d'une présence assidue du chercheur autorisant une certaine "participation » à la vie sociale (discontinue toutefois puisque je n'ai pas résidé dans les quartiers en question). L'enquête de Dûwîqa a été menée en deux lieux différents : Uhâyyîd et surtout Talâtât. J'ai réalisé dix entretiens dans la première zone. S'agissant du second site d'enquête, j'ai pu me rendre régulièrement, entre 2000 et 2002, dans une rue située près du marché, accueilli par une famille disposant d'une habitation à Dûwîqa mais résidant la plupart du temps à Darb al-Ahmar, quartier limitrophe (figure 1). J'ai également accompagné à de nombreuses reprises les différents membres de cette famille dans leurs pérégrinations en ville. D'autre part, afin de disposer d'une mesure statistique des déplacements quotidiens, j'ai eu recours aux données d'une enquête sur les mobilités recueillies par un bureau d'étude français (Systra) en charge de la rédaction d'un plan transport du Grand-Caire ${ }^{8}$.

\section{Personnalité d'un « quartier étendu »}

\section{Émergence d'un quartier aux marges de la ville}

Dûwîqa ${ }^{9}$ appartient à la première ceinture périphérique dont l'urbanisation s'inscrit dans la somme des modifications urbaines que connaît Le Caire depuis une trentaine d'années. Ces évolutions se caractérisent par un ralentissement de la croissance démographique ${ }^{10}$ accompagnée d'une redistribution des populations et des activités sur une superficie urbaine en extension [Denis, 1998 a]. Ce redéploiement démographique des quartiers centraux vers les périphéries est perceptible depuis les années soixantedix. Agnès Deboulet constate ainsi que les quinze qism-s du centre-ville ont nettement perdu en densité avec un solde négatif de 402721 personnes depuis 1976; parallèlement, les habitants des nouvelles périphéries apparaissent le plus souvent originaires de la capitale [Deboulet, $1991: 8-10]$.

\footnotetext{
7 Le terme fait référence non pas à une substance mais à une dimension des phénomènes sociaux prenant en compte une différence située et concrète [Appaduraï, 2001 : 42].

Les résultats sont présentés dans: Arab Republic of Egypt, Ministry of Transport, National Authority for Tunnels, Greater Cairo Public Transport Study, 6 vol., août 2000. L'enquête a porté sur un échantillon de 60000 personnes, âgées de plus de 6 ans en 1998, dans l'aire métropolitaine cairote (districts urbains uniquement).

La notion de quartier renvoie à des réalités d'échelles différentes, de la ruelle et de la rue aux ensembles réunis sous le même toponyme et inspirateurs d'une conscience territoriale pouvant enchâsser des zones de moindre familiarité. Ces derniers sont, dans le cas du Caire, des « quartiers étendus ». Ils correspondent en gros aux qism-s (districts) du maillage administratif de l'agglomération, lesquels dépassent couramment les 50000 habitants. Ils sont constitués de «quartiers intermédiaires » qui correspondent par la taille aux shiyakha-s (arrondissements) et les recoupent partiellement, et de " microquartiers » constitués par les habitations d'une ruelle et dont le nom historique est hara.

Le taux de croissance annuel de la population du Grand-Caire (Le Caire, Giza et Qalyûbiyya : 13,5 millions d'habitants en 1996) est passé de 2,7\% entre 1976 et 1986 à 1,6 \% entre 1986 et 1996 . Pour le seul gouvernorat du Caire (6,9 millions en 1996), le taux a baissé de 1,8\% à 1,1\% [Denis 1998 b (erratum 1999) : $16(71)]$.
} 
La dégradation extrême de nombreux immeubles centraux comme l'expulsion d'habitants devenus indésirables dans les bâtiments historiques provoquent une importante migration vers les nouvelles zones résidentielles de la première ceinture, dont Dûwîqa. En effet, depuis que la vieille ville est l'objet d'une redécouverte patrimoniale - elle est inscrite sur la liste du patrimoine mondial de l'Unesco depuis 1979 -, de nombreux squatters des édifices anciens sont délogés par les travaux de rénovation. Les "refuges" répondent ainsi à la nécessité de reloger les familles expulsées et de faire face aux situations d'urgence sanitaire. Ils sont considérés comme une solution temporaire aux difficultés rencontrées par des ménages paupérisés.

La mobilité résidentielle constitue donc un enjeu primordial pour les autorités qui souhaitent améliorer l'équilibre démographique de la ville et dédensifier les quartiers historiques mais aussi pour les habitants aux moyens économiques extrêmement limités. L'obtention d'un refuge permet aux familles pauvres de disposer d'un appartement de deux pièces alors qu'elles ne bénéficiaient bien souvent que d'une pièce unique. Elle offre également la possibilité, à terme, d'accéder à un logement social confortable et équipé, à un prix modique et bien en deçà de celui du marché.

Aucun refuge n'est plus attribué depuis l'an 2000. Avant cela, les démarches pour l'obtention d'un refuge prenaient entre une et trois années. Dans l'attente du nouveau logement, les familles à la rue étaient hébergées sous des tentes de l'armée à Bab alUzîr, dans le voisinage immédiat de la citadelle et à proximité de Minshât Nasîr. Les dossiers étaient déposés auprès du bureau du logement du gouvernorat du Caire. De nombreuses pièces devaient être produites dont le procès-verbal attestant de la destruction ou de l'insalubrité du logement précédemment occupé (notamment à la suite du tremblement de terre de 1992) et une déclaration de ressources. Toutefois, les critères d'attribution ne semblent pas avoir été clairement définis, les demandeurs ne maîtrisant ni les règles d'attribution, ni la procédure à suivre. Ils montaient leur dossier de façon improvisée, l'ensemble des pièces requises n'étant pas établi dès le départ. De fait, les démarches étaient très contraignantes et nécessitaient de se rendre dans différentes administrations (commissariat, affaires sociales, gouvernorat, poste de police, etc.). Elles supposaient également de solliciter des réseaux personnels afin de faciliter le traitement du dossier. Pour celui qui ne connaissait personne directement, quelques petits billets judicieusement distribués aux fonctionnaires aidaient à l'affaire. Cette petite corruption se révèle en général indispensable en accompagnement d'un dossier cheminant dans les arcanes administratifs. De même, il pouvait arriver que des candidats, jouant sur plusieurs tableaux, tentent d'émouvoir le responsable des attributions en présentant une situation familiale difficile, quitte, comme je l'ai vu faire, à traîner une épouse hospitalisée jusqu'au bureau du fonctionnaire pour qu'il constate la précarité de sa santé. Comme l'écrivent N. Beyhum et J.-C. David [1997 : 198], «le fonctionnement des pouvoirs ménage une multitude d'ouvertures et de moyens d'intervention, de négociation, de mises au point de compromis qui permettent de répartir les privilèges pour les rentabiliser, mais aussi de gouverner à la fois par l'administration et les rouages officiels et par les réseaux »".

Suivant une voie parallèle à ce circuit administratif négocié, d'autres habitants se sont appropriés un refuge en l'occupant, puis ont réussi au bout de plusieurs années à officialiser leur présence et à être considérés comme des bénéficiaires à part entière. Enfin, un petit marché immobilier informel s'est greffé sur le parc public du fait des bénéficiaires qui mettent en vente leur logement, au coût de cinq à quinze mille livres 
égyptiennes selon l'emplacement, ou bien le louent pour un loyer mensuel compris entre cent et deux cents livres.

Dûwîqa fait aujourd'hui partie de Mahagar, l'un des sept arrondissements de Minshât Nasîr (figure 1) qui abrite des habitations édifiées en marge des règlements administratifs. Cette vaste cité d'habitat «informel» est désormais intégrée dans le maillage administratif cairote, mais son développement et son avenir demeurent problématiques. Les données du recensement de 1996 ont largement sous-estimé les populations de Dûwîqa et plus généralement de Minshât Nasîr en affectant respectivement 1110 et 168425 habitants à ces deux entités. Ces valeurs peuvent être multipliées par 100 pour la première et par quatre pour la seconde pour obtenir une image plus réaliste de leur poids démographique. La population de Dûwîqa était estimée, en effet, à 100000 habitants (exactement 101340 en 2001, selon le comptage des "Autorités de restauration »: Gouvernorat du Caire, Ministère du logement, des services publics et des communautés urbaines, GOPP, GTZ) avant que ne soient entamés, à la fin de l'année 2001, la destruction d'une petite partie des logements et le déplacement des populations vers des habitations pérennisées ${ }^{11}$.

\section{Figure 1 - Carte du Grand-Caire (gouvernorats et qism-s)}

Figure 2 - Shiyakha-s de Minshât Nasîr

\section{(insérer fichiers EPS)}

\section{Aménagement des espaces}

Dûwîqa est un espace bien délimité et encadré par des édifices gouvernementaux. En effet, le siège de l'administration du district de Minshât Nasîr et la caserne de police sont disposés de part et d'autre de la seule entrée carrossable. Au sommet de la colline sur laquelle s'étage le quartier étendu se trouve la partie aménagée dite al-Harrafiyyin (« les artisans ») dont les méthodiques alignements d'immeubles de plusieurs étages abritent à leur pied les ateliers, principalement de mécanique automobile, de carrosserie et peinture, refoulés du centre du Caire pour cause de pollution.

Le quartier se compose de trois parties. Chaque type de constructions occupe une portion spécifique de la colline. Les unités sans étage sont disposées au pied, le long de l'avenue Tayarân; les immeubles de un et deux étages les surmontent. Chacun de ces habitats a inspiré un toponyme en rapport à son implantation et à sa taille, respectivement : Uhayyid, pour les constructions de plain-pied, Itninât, pour celles à deux niveaux et Talâtât, pour celles à trois niveaux. Cette dernière partie est ainsi constituée de plusieurs îlots ménageant des rues perpendiculaires à l'avenue principale,

11 Ces destructions interviennent dans la partie Uhâyyîd, bordant l'avenue Tayarân. J'estime entre 3000 et 4000 le nombre des personnes concernées au moment de la rédaction de cet article. Les habitants sont relogés dans la cité d'habitat social Dûwîqa al-Gadîda. La destruction était prévue de longue date du fait de la mauvaise qualité des refuges; les nouveaux logements sont d'un meilleur standing et par conséquent plus coûteux : l'épargne préalable de cette formule de location-vente est l'équivalent de 200 à 250 euros, le loyer mensuel de 12 à 14 euros, et l'accession à la propriété se fait entre 15 et 25 ans. À l'inverse, les refuges sont quasiment gratuits (l'équivalent d'environ un euro par mois charges, eau et électricité comprises, dont les locataires ne s'acquittent d'ailleurs que rarement). Le programme de relogement devrait s'étaler sur plusieurs années au rythme des constructions de logement sociaux. 
« Sûq at-Talâtât », sur laquelle se tient un marché permanent (cf. hors-texte). Un espace resté vierge de toute construction est appelé "le stade» (hors-texte). Il est utilisé comme terrain de football par les jeunes depuis que les poubelles qui l'encombraient ont été précipitées en bas de la colline par un engin spécialement affrété pour cette tâche par les habitants.

Les bâtiments livrés par le gouvernorat ont fait l'objet de plusieurs types de rajouts à l'instigation de leurs occupants. Pour les maisons individuelles, un mur surmonté parfois d'un grillage vient enclore une partie de l'espace de la rue, la privatisant et l'intégrant dans le domaine domestique (cf. hors-texte). Plus loin, pour ceux qui disposaient de moins de moyens, l'appropriation de la portion de rue devant l'appartement se manifeste par un muret prolongeant les limites domestiques sur un ou deux mètres (hors-texte). Les appartements situés en rez-de-chaussée des immeubles ont en outre été agrandis d'une pièce gagnée sur l'extérieur, qui vient rompre l'alignement des façades. Enfin, quelques modestes constructions érigées en toute discrétion sont destinées à abriter les membres d'une famille désormais trop à l'étroit dans son « refuge ». Les années passant, beaucoup de personnes ont en effet installé de petits ateliers de menuiserie, de fabrication de mobilier, de modestes entrepôts de marchandises destinées à la revente, etc.

Ces extensions informelles ont connu une intensification à "Tâlâtât 》 depuis l'été 2002. Pourtant, chacun a pu observer les destructions opérées ailleurs dans le quartier. Mais considérant la lenteur des travaux de construction des logements destinés à remplacer les refuges à Duwîqâ al-Gadîda - et la suppression d'une partie de l'aide économique américaine qui l'expliquerait ${ }^{12}$-, les habitants font l'analyse qu'il s'écoulera beaucoup de temps avant la livraison des prochaines tranches de logements sociaux. Ils préfèrent donc investir dans ce qu'ils possèdent déjà plutôt que d'attendre un hypothétique relogement. Le prix d'un refuge à Dûwîqa a d'ailleurs doublé en quelques mois sur le marché noir, signe que les habitants ne considèrent pas que cette zone est menacée à cours ou moyen terme.

Habitat social et extensions informelles s'enchevêtrent donc à Dûwîqa ${ }^{13}$. L'appropriation de l'espace dont le quartier est le théâtre passe par la constitution d'une société de proximité dans laquelle normes sociales, cultures urbaines, mode et territoire de vie sont étroitement liés. Le voisinage n'y désigne pas seulement une proximité physique en même temps qu'une familiarité entretenue entre personnes partageant un même espace de résidence [McKenzie, 1990: 235]; il constitue aussi le socle d'une organisation sociale à base territoriale.

\section{La vie sociale : formation d'une communauté de voisinage}

\section{Installation ; intégration}

Dûwîqa fut cependant d'abord un repoussoir plus que d'emblée un lieu propice au développement d'une vie communautaire. Jusqu'au milieu des années quatre-vingt, il

12 À la suite de la condamnation par un tribunal du Caire d'un citoyen américano-égyptien. La rumeur attribue cette suppression à la position égyptienne qui s'opposerait à une guerre contre l'Irak. Comme on le verra, ce type d'information circule dans les micro-arènes de sociabilité disséminées dans le quartier.

${ }_{13} 45 \%$ de la population urbaine égyptienne vivraient en habitat informel [Bayat, Denis, 2000]. Sur ces zones urbaines, voir plus précisément la thèse d'Agnès Deboulet [1994]. 
était un repère de malfrats chassés de la vieille ville (notamment de Batniyya à Darb alAhmar, lieu important de la vente de haschich). Avant l'entrée en service de la caserne de police au début des années quatre-vingt-dix, les rixes et conflits y étaient fréquents et pouvaient prendre des proportions importantes. L'ensemble de Minshât Nasîr dépendait alors du poste de Gamâliyya, trop éloignée pour une intervention efficace. De nombreux bénéficiaires de refuges ont donc quitté rapidement la zone du fait du climat de violence qui régnait alors. Ainsi que le rappelle Hammatu, sur place depuis plus de vingt ans : « Nous avions tous peur en partant travailler le matin de constater le soir en rentrant que notre femme ou un de nos enfants avait été blessé à la suite d'une querelle. De nos jours, les altercations ne sont plus aussi dures et il n'y a plus de bagarres graves. »

Pour ceux qui sont restés, faute de mieux, l'installation fut difficile comme en témoigne cette habitante de la première heure à Talâtât : " À Dûwîqa, il n'y a pas de mawlid (fête commémorative d'un saint ou d'une sainte); c'est de l'habitat de montagne, il n'y a pas de cheikh - en référence à la topographie du quartier et au fait qu'on n'y trouve pas de tombeau de saint -, c'est impossible. Ici c'est simple, il y a un souk et une station de transport. Avant, c'était vide et nous avions peur, nous arrivions de quartiers très peuplés. »

Les anciens habitants jouent un rôle important dans l'accueil et l'intégration des nouveaux arrivants. Cette intégration se joue en plusieurs micro-arènes disséminées dans le quartier, et notamment à l'échelle d'une portion de rue rassemblant une vingtaine de ménages. Elle se manifeste tout d'abord par divers services et par une entraide féminine autour des questions domestiques. Les invitations à participer aux veillées masculines et féminines se multiplient. Celles-ci représentent un élément clé des sociabilités dans le quartier. Les hommes, par groupe d'affinités, se retrouvent chez l'un ou l'autre des voisins, parfois au café. Les rencontres commentent les dernières rumeurs comme les "événements de la ville», pour reprendre le titre d'une émission très suivie. Les postes de télévision, installés devant les maisons dès que le temps se réchauffe, offrent d'ailleurs l'occasion de petits regroupements le temps de regarder, avec une attention inégale, informations et feuilletons égyptiens. Structurant les premières relations sociales et distribuant de la familiarité entre les hommes, cette sociabilité de proximité est un moment privilégié d'échange d'idées et de représentations du monde.

Pour les nouveaux arrivants en provenance de quartiers où ce type de sociabilité aujourd'hui a peu ou prou disparu, l'étonnement est donc grand. Père de famille originaire de Darb al-Ahmar, Ahmad a été surpris par cette vie communautaire lors de son arrivée dans le quartier. Il note ainsi que les habitants de Dûwîqa « prennent soin les uns des autres et vivent porte ouverte. Mais quand ils s'engueulent, attention! Ici (à Darb al-Ahmar), il y a un peu de civilité mais là-bas (à Dûwîqa), le niveau est moins élevé encore. Pourtant, ils sont très hospitaliers. Ils invitent à manger, partagent ce qu'ils ont. Les jeunes ont 50 piastres pour la journée et encore. Ils les jouent en faisant des paris sur le terrain de foot derrière notre maison et s'engueulent souvent. Les femmes aussi se battent, se tirent par les cheveux [...]. Quand on est arrivés, on a été très bien accueillis. » Son épouse de renchérir : «En arrivant, la voisine m'a demandé si j'avais une machine à laver non automatique. Elle m'a proposé d'utiliser la sienne. » Mais cette femme laisse fenêtres et portes fermées car elle n'est pas habituée au quartier. On lui demandera alors si elle a fait quelque chose, si elle a un problème. Le désir d'une société "lisible» en totalité s'exprime dans l'ouverture des espaces domestiques et notamment de la porte d'entrée de la maison, au sein du voisinage. 


\section{Ouverture de l'habitation, ouverture aux autres}

À la lisibilité du social répond le caractère «lisse » de l'espace : de la rue ou de la ruelle jusqu'à l'intérieur de la maison, aucune aspérité ne vient interrompre le cheminement, si ce n'est un simple rideau destiné à voiler l'intimité domestique. En somme, une porte fermée est suspecte ; il ne s'agit pas d'épier les gens chez eux mais plutôt de se donner la simple possibilité d'un contrôle. Laisser sa porte ouverte montre son acceptation du regard des autres dans le quartier. Si l'on n'a rien à cacher, pourquoi alors s'enfermer et se dérober à la sagacité du voisinage ?

Montrer sa disponibilité, c'est au contraire assurer du caractère inoffensif de sa présence. En effet, la continuité ainsi instaurée entre la ruelle et la sphère domestique, l'interpénétration entre ces deux domaines, viennent renforcer le sentiment communautaire et garantir les bonnes dispositions des uns envers les autres. L'ouverture de la maison répondrait ainsi aux nécessités d'une « pacification » sociale ${ }^{14}$, laquelle s'exerce avec d'autant plus de force que le quartier est récent et que les départs et arrivées y sont nombreux. À Dûwîqa, "l'autre », c'est-à-dire le nouvel arrivant, est rapidement converti en « semblable » par son insertion dans des cercles des relations de proximité.

Le contrôle social s'exerce ainsi sur la moralité des membres, et celui qui déroge aux dispositions communes est rappelé à l'ordre par les injonctions du voisinage. Appuyons-nous sur un exemple : un homme qui amenait chez lui des prostituées est publiquement pris à partie par une voisine qui lui reproche son comportement et son influence négative sur le quartier. L'altercation gagna en intensité quand les autres femmes de l'immeuble vinrent soutenir les doléances de la première. L'épouse de l'homme réprimandé intervint alors pour le défendre, en affirmant son plein accord avec le comportement de son époux, que c'était même elle qui choisissait ses amantes, et que « cela ne les regardait pas de toute façon ». Le mari volage, quant à lui, a considéré que cela n'était que «jalousie d'insatisfaites » de la part de ses accusatrices. Finalement, après des explications de plus en plus vives, la dispute prit fin grâce à l'intervention d'une femme plus âgée disposant d'une forte autorité dans la rue, et avec la promesse que ce comportement licencieux cessera. De telles médiations s'observent lors de chacun des petits conflits qui régulièrement secouent la communauté de voisinage.

Ces querelles ont de multiples mobiles ayant trait à la vie commune: des associations économiques mises en péril, des bagarres d'enfants relayées par les parents, le manque de respect à une femme, etc. La violence est la plupart du temps contenue et des procédures de régulation des comportements sont à l'œuvre lors de chaque conflit : par exemple un tiers interviendra toujours pour séparer et calmer les belligérants. Ce contrôle de la violence que l'on voit à toute occasion s'exercer, malgré l'emphase et le désordre apparents, dénote somme toute une société urbaine policée.

Les conflits demeurent d'ailleurs individuels ou cantonnés à l'échelle d'une famille. Les solidarités élargies, comme celles qui unissaient les membres de grandes familles originaires de Haute-Égypte, dans le Imbaba (vaste quartier informel de l'ouest du Caire) des années quatre-vingt-dix [Haenni, 2001 : 173-177], n'existent pas à Dûwîqa du fait de la dispersion des familles et de l'autonomie qu'elles ont prise à l'égard de leurs cercles d'appartenance d'origine. L'ancrage citadin des habitants de Dûwîqa

14 «La production d'un voisinage est intrinsèquement colonisant, au sens qu'elle implique l'affirmation d'un pouvoir socialement et (souvent rituellement) organisé sur des lieux et des installations perçus comme potentiellement chaotiques ou rebelles » [Appaduraï, $2001: 254$ ]. 
remonte en effet à plusieurs générations. Dans le même ordre d'idée, n'existent pas non plus dans le quartier ces conseils de représentants de grandes familles qui, à Imbaba par exemple, ont vocation à apaiser les discordes.

La surprise de l'homme qui avait subi l'opprobre d'une partie des habitants de la rue $\mathrm{du}$ fait de son comportement, en revanche, met en relief la force du contrôle social à Dûwîqa. Arrivée récemment dans le quartier, cette personne s'étonnait d'une telle immixtion dans sa vie privée qui ne s'était jamais produite dans la vieille ville d'où il était originaire. La vie collective adossée à l'espace de résidence connaît en effet, dans les quartiers historiques où elle était pourtant le plus développée, un certain recul du fait d'un double phénomène. Un transfert de fonction y est d'abord à l'œuvre depuis les années soixante-dix. Outre le remplacement de logements par des ateliers, et conséquemment l'émigration d'anciens habitants, ces nouvelles activités manufacturières ouvrent l'espace intime des hara-s (micro-quartiers historiques) à de nouveaux flux d'anonymes qui, par leur simple présence, interdisent la reconduction de pratiques d'usage communautaire des rues et des ruelles : « À Darb al-Ahmar, on croise beaucoup de gens que l'on ne connait pas, venus visiter un des ateliers; tandis que si je vois un inconnu à Dûwîqa, c'est qu'il s'est perdu. » (Ahmad, Dûwîqa) La seconde évolution générale tient à la diffusion de comportements individualistes qui conduit de plus en plus de ménages à s'extraire partiellement des relations avec le voisinage.

La vie sociale plus ouverte à Dûwîqa ne se traduit pas uniquement par le rappel de prescriptions morales et par la force du contrôle social. Il ressort de nombreux discours entendus dans la rue que « les habitants se sentent bien ». Leur sociabilité et l'ambiance communautaire du quartier sont mises en exergue : "On y ressent la chaleur du hara » (Ahmad, Dûwîqa). La froideur et la pauvreté des relations sociales entre voisins des quartiers bourgeois sont opposées à l'animation des rues populaires. Faut-il accréditer l'idée qu'il existe "dans la vie des taudis, des compensations que les habitants des banlieues résidentielles n'imaginent pas » [Park, cité par Grafmeyer, Joseph, 1990 : 17] ? Retenons que les habitants que j'ai fréquentés à Talâtât se sentent « chez eux ", ainsi que le dit une résidente de la rue étudiée : "On a construit nos vies ici, il y a du travail et des écoles maintenant. Il n'y a pas d'autre lieu, ici, c'est notre quartier. »

Soulignons enfin que cette vie sociale peut se révéler contraignante et certains, à Dûwîqa comme ailleurs, préfèrent sortir le plus possible de la pression communautaire, à l'instar de jeunes mariés qui venaient de louer un appartement dans la ruelle et dont on savait finalement bien peu de chose. Les matrones regroupées sur le pas des portes ont ainsi toute latitude pour imaginer et commenter la vie d'autrui en buvant un thé sirupeux. C'est donc en terme de tendance qu'il faut raisonner pour comprendre le relief particulier du quartier sans pour autant enfermer ses habitants dans les cercles du voisinage, ni faire de l'ancrage territorial l'unique ressort de leur vie sociale. Certains tentent malgré tout, comme on l'a vu, de rester "porte fermée ». Dans tous les cas, l'ancrage n'exclut pas de tisser des liens avec d'autres places, d'autres gens et d'autres cultures urbaines, professionnelles notamment, comme en témoigne l'intensité des déplacements.

\section{Mobilités quotidiennes et relations des habitants à la ville}


"C'est la pauvreté qui fait bouger la ville»

Les résultats de l'enquête " ménages-transports » (Systra, 1998) dont nous disposons à propos des mobilités quotidiennes doivent être utilisés avec prudence. En effet, il s'agit d'une mesure par échantillonnage, dont les résultats ont été ensuite rapportés à la population totale telle qu'elle est décrite par le recensement de 1996. Or, comme on l'a dit, les effectifs du district de Minshât Nasîr et de l'arrondissement de Mahagar auquel appartient Dûwîqa ont été largement sous-évalués par ce recensement. C'est donc plus un ordre d'idée qu'il faut retenir qu'un reflet précis de la réalité des déplacements des habitants. L'enquête permet toutefois de donner une assise quantitative aux observations effectuées lors de la fréquentation du quartier. Y sont dénombrés les déplacements effectués en une journée à partir du district de Minshât Nasîr. Un déplacement correspond à un trajet quel qu'il soit, dans l'arrondissement de résidence ou depuis ce dernier en direction d'un autre arrondissement.

Figure 3 - Nombre de déplacements par jour et par personne

\begin{tabular}{|c|c|c|}
\hline Localisation des habitants & Hommes & Femmes \\
\hline Minshât Nasîr & 2,3 & 2,0 \\
\hline Moyenne Grand-Caire ${ }^{*}$ & 1,7 & 1,1 \\
\hline
\end{tabular}

Figure 4 - Tableau des déplacements quotidiens depuis Minshât Nasîr vers les autres districts du Grand-Caire

\begin{tabular}{lcccc}
\hline Qism-s (districts) & Hommes & $\mathbf{\%}$ & Femmes & \% \\
Minshât Nasîr & 109947 & 53,1 & 71812 & 67,6 \\
Vieille ville dont : & 23725 & 11,5 & 12831 & 12,0 \\
- Darb al-Ahmar & 9453 & 4,6 & 3430 & 3,2 \\
- Gamâliyya & 12219 & 5,9 & 9046 & 8,5 \\
Qism-s centraux & 27710 & 13,4 & 7958 & 7,5 \\
dont : & & & & \\
- al-Sâhil & 11350 & 5,5 & 5334 & 5,0 \\
- al-Wâylî & 11325 & 5,5 & 0 & 0 \\
$\begin{array}{l}\text { Autres qism-s du } \\
\text { gouvernorat du Caire }\end{array}$ & 36743 & 17,7 & 11448 & 10,8 \\
$\begin{array}{l}\text { Autres gouvernorats } \\
\text { région métropolitaine }\end{array}$ & 8974 & 4,3 & 2225 & 2,1 \\
$\begin{array}{l}\text { Total } \\
\text { ource : enquête « Ménages-transports », Systra, }\end{array}$ & 1998. & & \\
\end{tabular}

La tendance générale, depuis une trentaine d'années, est à l'accroissement des mobilités intra-urbaines. Elle est encore accentuée pour les habitants de Minshât Nasîr qui se déplacent davantage que ceux des autres districts de la ville, les hommes comme les femmes. Par ailleurs, les déplacements des femmes sont plus liés aux espaces proches que ceux des hommes comme le montre l'écart des pourcentages portant sur l'intérieur du district. Les femmes se déplacent par ailleurs un peu moins que les hommes (figure 3).

Par rapport aux autres qism-s cairotes, Minshât Nasîr constitue un district plutôt moins ouvert sur l'environnement urbain, à l'instar de Darb al-Ahmar avec lequel il 
partage le fait que les mobilités sont majoritairement locales. Or, en moyenne dans l'agglomération cairote, seul un tiers des déplacements sont internes au district considéré, les autres se faisant en direction d'un autre district de la ville [Barge, Puig, à paraître]. On peut faire l'hypothèse que beaucoup de ces déplacements courts sont liés en fait à un emploi sur place, dans les ateliers de Harrafiyyin notamment, mais également dans les commerces et les diverses activités économiques, pour la plupart informelles, qui se sont développées dans les rues de Dûwîqa.

Les déplacements vers les quartiers de la vieille ville (Darb al-Ahmar, Gamâliyya et dans une moindre mesure Bab Sha-ariyya) demeurent importants (12\%). Cela s'explique tout d'abord par le fait qu'il s'agit de zones limitrophes dans lesquelles on trouve de petits ateliers pourvoyeurs d'emploi et d'importants marchés de vêtements. Beaucoup d'habitants de Dûwîqa en sont au demeurant originaires et s'y rendent régulièrement pour des visites familiales (le principal motif des déplacements de femmes vers le qism de Gamâliyya). Au total, après les déplacements internes au qism de Minshât Nasîr, le centre ancien comprenant le vieux Caire (Fustât, création du VII ${ }^{\mathrm{e}}$ siècle), la vieille ville, et le centre actuel constitué dans la seconde moitié du XIX ${ }^{\mathrm{e}}$ siècle - 12 qism-s sur les 38 que compte le gouvernorat du Caire - draine un quart des déplacements.

Si l'on prend le cas de Darb al-Ahmar, on note que $12,2 \%$ des personnes se déplaçant en direction de ce qism sont originaires de Minshât Nasîr et qu'elles contribuent pour $14,8 \%$ aux déplacements totaux pour raison de travail à l'intérieur de cette portion de la vieille ville [Barge, Puig, à paraître]. Al-Waylî et al-Sâhîl concentrent quant à eux deux une petite partie des déplacements quotidiens enregistrés $(5,5 \%$ chacun). Ces trajets rendent compte de même des flux de travail vers les nombreuses petites industries et ateliers de ces deux arrondissements.

Ainsi, la mesure des mobilités à Minshât Nasîr présente-t-elle un tableau d'apparence contradictoire. Les déplacements quotidiens par personne y sont plus nombreux que dans la moyenne de la ville, mais ils s'inscrivent en majorité dans les frontières du qism. Si ces mobilités quotidiennes sont dans une large mesure "contraintes" par les conditions de vie - ce qu'un habitant de Dûwîqa exprimait par : "c'est la pauvreté qui fait bouger la ville »-, elles signalent la présence des populations les moins favorisés dans les différents lieux de la ville.

\section{Itinéraires esquissés}

Les déplacements hors du qism sont liés à deux réalités : soit on ne trouve pas dans le quartier les équipements nécessaires, soit on est amené à se déplacer pour se rendre sur le lieu de travail, organiser quelques menus trafics ou entretenir le réseau relationnel. Pour cela, il est essentiel de savoir se diriger dans la ville, d'en bien connaître la géographie. Ce thème fut un élément redondant de nos discussions avec les habitants de Dûwîqa.

La récurrence avec laquelle était évoqué le thème des transports, par exemple, témoigne de l'importance accordée par les habitants à l'accessibilité des différents lieux de la ville, notamment ceux qu'ils pratiquent régulièrement, voire quotidiennement. Au demeurant, le choix de Dûwîqa comme quartier d'accueil a souvent été lié à sa proximité des quartiers centraux et des anciens lieux de résidence. D'autres sites cairotes accueillent en effet des «refuges temporaires », mais ils sont plus éloignés. Nombre d'habitants de la proche périphérie sont liés à la vieille ville du fait de leur histoire personnelle et familiale, ce qui ne leur interdit pas de fréquenter également le 
centre-ville "européen». Ils cherchent, comme ceux des quartiers populaires en général, à multiplier les contacts et à profiter des ambiances urbaines, pratiques qui impliquent de nombreux déplacements.

Beaucoup conservent une possibilité d'hébergement dans le quartier d'origine. Pour les plus "nantis», qui bénéficient encore d'un appartement dans la vieille ville, le refuge alloué constitue un investissement pour l'avenir plus qu'une solution à un problème aigu de logement. Ahmad, par exemple, destine son refuge à son fils, né en 1993, qui pourra ainsi bénéficier d'un logement... après son mariage. Contrairement à Ahmad qui vient un peu en "touriste » à Dûwîqa, selon l'expression de l'un de ses voisins, Mahmûd a installé sa famille dans le refuge tout en conservant un autre logement dans Minshât Nasîr pour son usage personnel. Il s'y rend régulièrement, car il maintient sur place un petit réseau de connaissances professionnelles qui s'emploient à la tâche sur de petits chantiers (peinture, maçonnerie, plomberie, électricité, etc.). Il arrive également à Mahmûd de se rendre dans la vieille ville pour y rencontrer des amis. Les fêtes de mariage représentent notamment des moments privilégiés de préservation des anciennes sociabilités, les habitants de Dûwîqa étant régulièrement conviés aux noces de relations résidant dans les quartiers d'origine. On pourrait multiplier à l'envie les exemples de connexions entre Dûwîqa et les quartiers de la vieille ville, ce dont l'examen quantitatif des déplacements quotidiens ne rend pas bien compte à lui seul.

La société qui se dessine à Dûwîqa conjugue ainsi intimité sociale et mobilité vers les autres lieux de la ville. Le nouvel espace résidentiel a été constitué par les habitants en un lieu de vie sociale intense autorisant à parler à son propos de "société de proximité », ce qui n'enlève rien à son caractère citadin.

\section{À Dûwîqa, une société de proximité}

Création réglementaire provisoire destinée à héberger une population pauvre ne disposant pas de moyens de se maintenir dans leur quartier d'origine, la cité de Dûwîqa est devenue une réalité résidentielle et sociale plus durable exposée toutefois en certains lieux aux opérations de relogement entamées en 2001. Grâce à une vie sociale intense et intégrative, les habitants sont désormais enracinés dans une communauté à l'échelle du micro-quartier. Ce dernier est, en effet, un lieu majeur de socialisation et de prescription normative. Il représente un espace de contrôle moral et social des uns sur les autres, la proximité (densité physique comme proximité culturelle, économique et sociale) étant l'opérateur privilégié de cette relation entre le social et le spatial.

Aussi est-il possible de qualifier l'organisation collective qui prévaut à Dûwîqa, et dans les quartiers partageant ce type de disposition, de société de proximité. Le voisinage et la localité y sont étroitement articulés. Car si le premier est une forme sociale réalisée dans une organisation comprenant l'ensemble des dispositions matérielles et morales découlant de la proximité, la localité est une qualité phénoménologique de cette vie sociale [Appaduraï, 2001 : 247]. Dans ce « local », que figure le quartier, prend place un sentiment d'appartenance collective, de communauté de comportements et de vision partagée du monde.

Ce sentiment n'exclut pas d'autres ancrages, ce dont témoigne l'importance des mobilités. Le quartier d'origine, le lieu de travail et, de façon plus labile, le centre-ville avec lequel se maintient une certaine familiarité, vécue selon le sexe et la génération, sont autant de point de fixation permettant une pratique diversifiée des espaces urbains. C'est l'ensemble de ces points et des trajets les associant qui trame les «attitudes citadines ». Celles-ci comprennent tout à la fois les conceptions de la vie dans le 
quartier, les représentations de l'altérité (les autres cultures urbaines), les modes d'accès aux ressources concentrées dans la ville (transports urbains, relation aux administrations, possibilités d'emplois, sorties ludiques, etc.) et les relations aux espaces publics.

\section{Bibliographie}

APPADURAÏ A. [2001], Après le colonialisme, les conséquences culturelles de la globalisation, Paris, Payot, $315 \mathrm{p}$.

BARGE C., PUIG N. [à paraître], «Les cadres des expériences urbaines : mobilités et territoires dans Le Caire contemporain », in P. Signoles (dir.), Gouvernances dans le monde arabe (titre provisoire).

Battain T., LABIB A. [1991], «Le Caire, Mégapole perçue par ses habitants », Égypte - Monde arabe, $5: 19-41$.

Bayat A., Denis E. [2002], «Who is Afraid of Ashwaiyyat? Urban Change and Politics in Egypt», Environment and Urbanization, 12 (2) : 185-199.

BEYHUM N., DAVID J.-C. [1997], « Du souk à la place, du citadin au citoyen. Espaces publics dans les villes arabes (au Moyen-Orient)», Sciences sociales et Phénomènes urbains dans le monde arabe, Casablanca, Fondation du roi Abdul-Aziz Al Saoud pour les sciences sociales : 193-202.

Deboulet A. [1991], Des quartiers centraux vers les périphéries spontanées. Éléments sur la mobilité résidentielle dans la région du Grand-Caire, Tours-Le Caire, Urbama-Cedej, 34 p.

Deboulet A. [1994], Vers un urbanisme d'émanation populaire, Compétences et réalisations des citadins. L'exemple du Caire, thèse de doctorat, Institut d'urbanisme de Paris, université Paris-XIICréteil, $547 \mathrm{p}$.

DENIS E. [1998 a], «Croissance urbaine et dynamique sociospatiale. Le Caire de 1950 à 1990 », L'Espace géographique, 2 : 129-142.

DENIS E. [1998 b], «Le Caire et l'Égypte à l'orée du XXI ${ }^{\mathrm{e}}$ siècle. Une métropole stabilisée dans un contexte de redéploiement de la croissance », Lettre d'information de l'OUCC, 48:4-17 (erratum, $49: 71)$.

DePaUle J.-Ch. [1990], «Le Caire : Emplois du temps, emplois de l'espace », Monde arabe/MaghrebMachrek, $127:$ 121-133.

GRAFMEYER Y., JOSEPH I. [1990], «La ville laboratoire et le milieu urbain », in L'École de Chicago. Naissance de l'écologie urbaine, Paris, Aubier, Champ urbain : 5-52.

HAENNI P. [2001], Banlieues indociles? Sur la politisation des quartiers périurbains du Caire, thèse de doctorat, IEP Paris, $511 \mathrm{p}$.

HANNERZ U. [1983], Explorer la ville, Éléments d'anthropologie urbaine, Paris, Minuit, 418 p.

JOSEPH I. [1996], «Les compétences de rassemblement de rassemblement, une ethnographie des lieux publics », Enquête. La ville des sciences sociales, $4:$ 107-122.

JOSEPH I. [1997], « Prises, réserves, épreuves », Communications, 65 : 131-138.

JOSEPH I. [1998], La Ville sans qualités, La Tour d'Aigues, l'Aube, 209 p.

MCKenZIE R.D. [1990], «Le voisinage, Une étude de la vie locale à Colombus, Ohio », in L'École de Chicago, op. cit. : 213-254.

Reconstruction Authorities, Gouvernorate of Cairo, Ministry of Housing, Utilities AND Urban Communities, GOPP, GTZ [2001], The Participatory Urban Development of Manshiyyat Nasser Project, $156 \mathrm{p}$. 